\title{
Oscillatory Singularity Behaviors Near Interface Crack Tip for Mode II of Orthotropic Bimaterial
}

\author{
Xiaomei Yang, Weiyang Yang, Junlin Li, and Xuexia Zhang \\ School of Applied Science, Taiyuan University of Science and Technology, Taiyuan 030024, China \\ Correspondence should be addressed to Xiaomei Yang; yxm001_1@126.com
}

Received 20 December 2012; Revised 18 May 2013; Accepted 22 May 2013

Academic Editor: Ray K. L. Su

Copyright (C) 2013 Xiaomei Yang et al. This is an open access article distributed under the Creative Commons Attribution License, which permits unrestricted use, distribution, and reproduction in any medium, provided the original work is properly cited.

\begin{abstract}
The fracture behaviors near the interface crack tip for mode II of orthotropic bimaterial are discussed. The oscillatory singularity fields are researched. The stress functions are chosen which contain twelve undetermined coefficients and an unknown singularity exponent. Based on the boundary conditions and linear independence, the system of twelve nonhomogeneous linear equations is derived. According to the condition for the system of nonhomogeneous linear equations which has a solution, the singularity exponent is determined. Total coefficients are found by means of successive elimination of the unknowns. The theoretical formulae of stress intensity factors and analytic solutions of stress field near the interface crack tip are obtained. The crack tip field is shown by figures.
\end{abstract}

\section{Introduction}

Many researchers have studied the singularity behavior near interface crack tip for isotropic, orthotropic, or anisotropic bimaterial. The method of eigenfunction expansions is used by Williams [1]. The complex function method is developed by Rice and Sih [2]. Erdogan [3] and England [4], present and research various interface crack problems. These academic authorities play a leading role in fracture mechanics for interface crack, and they have made the tremendous contribution. The subsequent papers are published one after another over several decades [5-16]. In this paper, the solution method is proposed to research the singularity behavior near interface crack tip for mode II of orthotropic bimaterial.

It can be seen that the following differences exist between this method and previous methods by comparison.

(1) The stress function contains twelve undetermined coefficients, rather than eight.

(2) The system of twelve nonhomogeneous linear equations is deduced based on the boundary conditions, rather than the system of eight homogeneous linear equations.

(3) The characteristic equation is found by using the condition for the system of nonhomogeneous linear equations that possess a solution, rather than being based on the condition for the system of homogeneous linear equations which has a nontrivial solution.

(4) In order to determine total coefficients, we only need to solve the system of nonhomogeneous linear equations, rather than to solve the system of homogeneous linear equations first, and then use the load conditions at infinity.

(5) The stress intensity factors are defined by right-hand limit and left-hand limit rather than by limit.

(6) The oscillatory singularity fields near interface crack tip of three orthotropic bimaterial are illustrated by the help of two tables and seven figures.

\section{Mechanical Model}

The plane $y>0$ is the upper orthotropic material $(j=1)$, and its elastic constants are $E_{11}, E_{12}, \nu_{11}$ and $\mu_{1}$. The plane $y<0$ is the lower orthotropic material $(j=2)$, and its elastic constants are $E_{21}, E_{22}, v_{21}$, and $\mu_{2} \cdot y=0,|x|<a$ is the crack surfaces, and $y=0,|x|>a$ is the bonded interface of two dissimilar materials. 


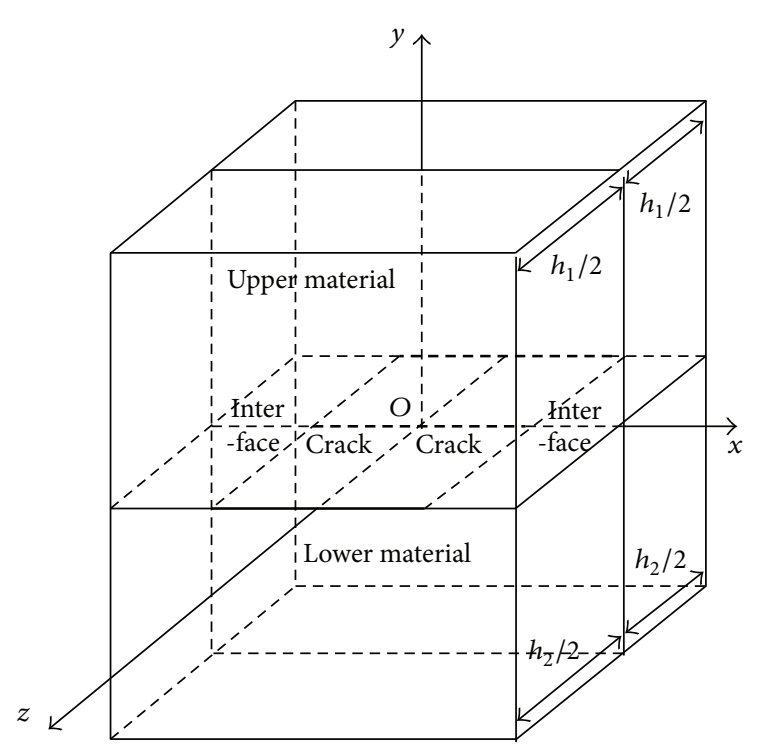

(a) Geometric state

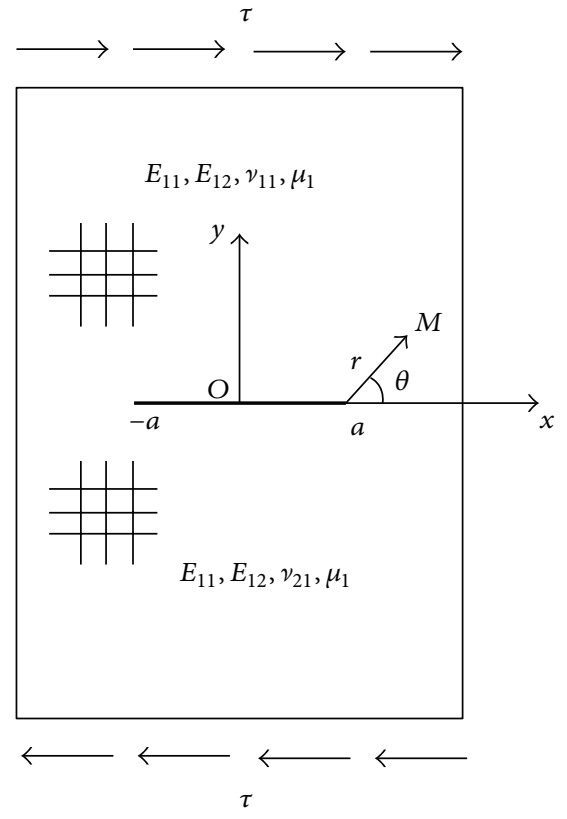

(b) Shear load

FIGURE 1: Interface crack for mode II of orthotropic bimaterial.

The stress functions $U_{j}(x, y),(j=1,2)$ satisfy the governing equations [16-20]:

$$
\begin{aligned}
\left(b_{22}\right)_{j} \frac{\partial^{4} U_{j}}{\partial x^{4}} & +\left[2\left(b_{12}\right)_{j}+\left(b_{66}\right)_{j}\right] \frac{\partial^{4} U_{j}}{\partial x^{2} \partial y^{2}} \\
& +\left(b_{11}\right)_{j} \frac{\partial^{4} U_{j}}{\partial y^{4}}=0, \quad(j=1,2) .
\end{aligned}
$$

The boundary conditions of the interface crack for mode II are as follows:

$$
\begin{gathered}
y=0, \quad|x|<a:\left(\sigma_{y}\right)_{1}=\left(\sigma_{y}\right)_{2}=0, \\
\left(\tau_{x y}\right)_{1}=\left(\tau_{x y}\right)_{2}=0, \\
y=0, \quad|x|>a:\left(\sigma_{y}\right)_{1}=\left(\sigma_{y}\right)_{2}, \quad\left(\tau_{x y}\right)_{1}=\left(\tau_{x y}\right)_{2}, \\
(u)_{1}=(u)_{2}, \quad(v)_{1}=(v)_{2}, \\
|y| \longrightarrow+\infty:\left(\sigma_{y}\right)_{1}=\left(\sigma_{y}\right)_{2}=0, \quad\left(\tau_{x y}\right)_{1}=\left(\tau_{x y}\right)_{2}=\tau .
\end{gathered}
$$

The geometric and load conditions for the investigated problem are given as shown in Figure 1.

\section{Stress Function}

The relationships between the stresses and the stress functions can be obtained as

$$
\begin{aligned}
\left(\sigma_{x}\right)_{j}= & \frac{\partial^{2} U_{j}}{\partial y^{2}} \\
=\sum_{k=1}^{2} \operatorname{Re}\{[ & \left.\left(A_{j k, \lambda}+i C_{j k, \lambda}\right)-i\left(B_{j k, \lambda}+i C_{j k, \lambda}\right)\right] \\
& \left.\times\left(-\beta_{j k}^{2}\right) U_{j k, \lambda}\left(z_{j k}\right)\right\}
\end{aligned}
$$

$$
\begin{aligned}
&\left(\sigma_{y}\right)_{j}= \frac{\partial^{2} U_{j}}{\partial x^{2}} \\
&=\sum_{k=1}^{2} \operatorname{Re}\left\{\left[\left(A_{j k, \lambda}+i C_{j k, \lambda}\right)\right.\right. \\
&\left.\left.\quad-i\left(B_{j k, \lambda}+i C_{j k, \lambda}\right)\right] U_{j k, \lambda}\left(z_{j k}\right)\right\},
\end{aligned}
$$

$$
\begin{aligned}
\left(\tau_{x y}\right)_{j}=-\frac{\partial^{2} U_{j}}{\partial x \partial y} & \\
=-\sum_{k=1}^{2} \operatorname{Re} & \left\{\left[\left(A_{j k, \lambda}+i C_{j k, \lambda}\right)-i\left(B_{j k, \lambda}+i C_{j k, \lambda}\right)\right]\right. \\
& \left.\times\left(i \beta_{j k}\right) U_{j k, \lambda}\left(z_{j k}\right)\right\}, \quad(j=1,2)
\end{aligned}
$$


in which the stress functions $U_{j}$ of right side of $(5 a)-(5 c)$ contain twelve undetermined coefficients $A_{j k, \lambda}, B_{j k, \lambda}, C_{j k, \lambda}$ $(j, k=1,2)$ and an unknown singularity exponent $\lambda$.

Considering the boundary conditions (2), (3), and (4), the functions $U_{j k, \lambda}\left(z_{j k}\right)$ of right side of $(5 \mathrm{a})-(5 \mathrm{c})$ can be chosen as

$$
U_{j k, \lambda}\left(z_{j k}\right)=\tau\left(1-\frac{a^{2}}{z_{j k}^{2}}\right)^{\lambda}=\tau \frac{z_{j k}^{-2 \lambda}}{\left(z_{j k}^{2}-a^{2}\right)^{-\lambda}}, \quad(j, k=1,2) .
$$

in which

$$
\begin{array}{r}
z_{j k}=x+s_{j k} y=x+i \beta_{j k} y, \quad(j, k=1,2) \\
z_{j k}-a=x-a+i \beta_{j k} y=r\left(\cos \theta+i \beta_{j k} \sin \theta\right), \\
(j, k=1,2) .
\end{array}
$$

From (5a)-(5c) and (6), an unknown number $\lambda$ is called singularity exponent, and sometimes it is also called eigenvalue.

\section{Singularity Exponent}

If the singularity exponent $\lambda$ is the complex number, the stress near the interface crack tip shows oscillatory singularity. We let $[1-16]$

$$
\lambda=-\frac{1}{2}+i \varepsilon .
$$

Substituting (9) into (6), it can be found as

$$
\begin{aligned}
U_{j k, \varepsilon}\left(z_{j k}\right) & =\tau\left(1-\frac{a^{2}}{z_{j k}^{2}}\right)^{-(1 / 2)+i \varepsilon} \\
& =\tau \frac{z_{j k}^{1-i 2 \varepsilon}}{\left(z_{j k}^{2}-a^{2}\right)^{(1 / 2)-i \varepsilon}}, \quad(j, k=1,2) .
\end{aligned}
$$

Equations (7) and (8) are substituted into (10), and we have

$$
\begin{aligned}
\left.U_{j k, \varepsilon}\left(z_{j k}\right)\right|_{y=0,|x|<a}= & e^{(-1)^{j} \varepsilon \pi} \frac{\tau x}{\sqrt{a^{2}-x^{2}}}(-1)^{j-1} \\
\times & {\left[\sin \left(2 \varepsilon \ln \frac{\sqrt{a^{2}-x^{2}}}{x}\right)\right.} \\
& \left.\quad-i \cos \left(2 \varepsilon \ln \frac{\sqrt{a^{2}-x^{2}}}{x}\right)\right] .
\end{aligned}
$$

at the crack surfaces.

Substituting (11), (5b), and (5c) into the boundary condition (2), by the help of the functions $\cos \left(2 \varepsilon \ln \left(\left(\sqrt{a^{2}-x^{2}}\right) / x\right)\right)$ and $\sin \left(2 \varepsilon \ln \left(\left(\sqrt{a^{2}-x^{2}}\right) / x\right)\right)$ which are linearly independent, the four homogeneous linear equations can be deduced as

$$
\begin{gathered}
A_{11, \varepsilon}+A_{12, \varepsilon}-B_{11, \varepsilon}-B_{12, \varepsilon}+2 C_{11, \varepsilon}+2 C_{12, \varepsilon}=0, \\
-A_{21, \varepsilon}-A_{22, \varepsilon}+B_{21, \varepsilon}+B_{22, \varepsilon}-2 C_{21, \varepsilon}-2 C_{22, \varepsilon}=0, \\
-\beta_{11} A_{11, \varepsilon}-\beta_{12} A_{12, \varepsilon}-\beta_{11} B_{11, \varepsilon}-\beta_{12} B_{12, \varepsilon}=0, \\
\beta_{21} A_{21, \varepsilon}+\beta_{22} A_{22, \varepsilon}+\beta_{21} B_{21, \varepsilon}+\beta_{22} B_{22, \varepsilon}=0 .
\end{gathered}
$$

Similarly, we can find other eight linear equations (12e) to (12l) which are omitted here. By $\left(\tau_{x y}\right)_{1}=\left(\tau_{x y}\right)_{2}=\tau$ in condition (4), two nonhomogeneous linear equations are contained in the eight linear equations.

The system of nonhomogeneous linear equations (12a) to (12l) is solved by eliminating the unknowns. To let the rank of the coefficient matrix $A_{\lambda}$ for the system (12a)-(12l) and the rank of the augmented matrix $\bar{A}_{\lambda}=\left(A_{\lambda}, b_{\mathrm{II}}\right)$ be equal, the rank must be 8 ; that is, $\operatorname{rank}\left(A_{\lambda}\right)=\operatorname{rank}\left(\bar{A}_{\lambda}\right)=8$. For this reason [21], the number $\varepsilon$ must satisfy the following characteristic equation:

$$
\left(e_{12}-f_{12}\right) f_{12}-4 g_{12} h_{12} \varepsilon^{2}=0
$$

in which bimaterial parameters are given as

$$
\begin{gathered}
e_{12}=\frac{1}{\mu_{1}}-\frac{1}{\mu_{2}}, \quad f_{12}=\frac{\beta_{11} \beta_{12}-v_{11}}{E_{11}}-\frac{\beta_{21} \beta_{22}-v_{21}}{E_{21}}, \\
g_{12}=\frac{\beta_{11}+\beta_{12}}{E_{11}}+\frac{\beta_{21}+\beta_{22}}{E_{21}}, \\
h_{12}=\frac{\beta_{11}+\beta_{12}}{E_{11}} \beta_{11} \beta_{12}+\frac{\beta_{21}+\beta_{22}}{E_{21}} \beta_{21} \beta_{22} .
\end{gathered}
$$

The singularity exponent $\lambda$ of (9) is a complex number, so $\varepsilon \neq 0$ in (13), and $e_{12} \neq 0, f_{12} \neq 0, g_{12}>0, h_{12}>0$ in (14a) and (14b) for determinate bimaterial. To the equation (13) has a solution, the discrimination rule can be deduced as

(*) $\mathrm{D}_{12}$ : if $e_{12}$ and $f_{12}$ in (14a) satisfy $e_{12}>0$ and $f_{12}>0$, then the negative root of (13) is the bielastic constant $\varepsilon$.

Substituting $\varepsilon$ solved by (13) into (9), the complex singularity exponent $\lambda$ can be obtained.

The system of eight equations which remained through sequence elimination is solved by means of the inverse sequence backsubstitution. We found all coefficients as follows:

$$
\begin{gathered}
A_{j k, \varepsilon}+C_{j k, \varepsilon}=(-1)^{k} \frac{f_{12}+2 \beta_{j k^{*}} g_{12} \varepsilon}{\left(\beta_{j 2}-\beta_{j 1}\right) f_{12}}, \\
B_{j k, \varepsilon}-C_{j k, \varepsilon}=(-1)^{k-1} \frac{f_{12}-2 \beta_{j k^{*}} g_{12} \varepsilon}{\left(\beta_{j 2}-\beta_{j 1}\right) f_{12}}, \quad(j, k=1,2) .
\end{gathered}
$$

in which $k=1, k^{*}=2 ; k=2, k^{*}=1$. By (15a) and (15b), the stress functions $U_{j}$ of the right side of $(5 a)-(5 c)$ contain practically eight coefficients. 


\section{Stress Intensity Factors}

Considering the stress expressions (5b) and (5c) and also the load condition in (4), the stress intensity factors are defined as

$$
\begin{aligned}
& \left(k_{2}^{+}\right)_{j}=-\sum_{k=1}^{2} \lim _{z_{j k} \rightarrow a^{+}} \operatorname{Re}\left\{\left(2 \pi\left|z_{j k}-a\right|\right)^{(1 / 2)-i \varepsilon}\right. \\
& \times\left[\left(A_{j k, \varepsilon}+C_{j k, \varepsilon}\right)\right. \\
& \left.-i\left(B_{j k, \varepsilon}-C_{j k, \varepsilon}\right)\right] \\
& \left.\times\left(i \beta_{j k}\right) U_{j k, \varepsilon}\left(z_{j k}\right)\right\}, \\
& \left(k_{2}^{-}\right)_{j}=-\sum_{k=1}^{2} \lim _{z_{j k} \rightarrow a^{-}} \operatorname{Re}\left\{\left(2 \pi\left|z_{j k}-a\right|\right)^{(1 / 2)-i \varepsilon}\right. \\
& \times\left[\left(A_{j k, \varepsilon}+C_{j k, \varepsilon}\right)\right. \\
& \left.-i\left(B_{j k, \varepsilon}-C_{j k, \varepsilon}\right)\right] \\
& \times(-1)^{j-1}(2 \varepsilon-i)\left(i \beta_{j k}\right) \\
& \left.\times U_{j k, \varepsilon}\left(z_{j k}\right)\right\}, \quad(j=1,2) \text {, } \\
& k_{2}=\frac{k_{2}^{+}-k_{2}^{-}}{2}=\frac{1}{2} \sum_{j=1}^{2} \frac{\left(k_{2}^{+}\right)_{j}}{2}-\frac{1}{2} \sum_{j=1}^{2} \frac{\left(k_{2}^{-}\right)_{j}}{2}, \\
& \left(k_{1}^{+}\right)_{j}=\sum_{k=1}^{2} \lim _{z_{j k} \rightarrow a^{+}} \operatorname{Re}\left\{\left(2 \pi\left|z_{j k}-a\right|\right)^{(1 / 2)-i \varepsilon}\right. \\
& \times\left[\left(A_{j k, \varepsilon}+C_{j k, \varepsilon}\right)-i\left(B_{j k, \varepsilon}-C_{j k, \varepsilon}\right)\right] \\
& \left.\times U_{j k, \varepsilon}\left(z_{j k}\right)\right\},
\end{aligned}
$$$$
\left(k_{1}^{-}\right)_{j}=\sum_{k=1}^{2} \lim _{z_{j k} \rightarrow a^{-}} \operatorname{Re}\left\{\left(2 \pi\left|z_{j k}-a\right|\right)^{(1 / 2)-i \varepsilon}\right.
$$$$
\times\left[\left(A_{j k, \varepsilon}+C_{j k, \varepsilon}\right)-i\left(B_{j k, \varepsilon}-C_{j k, \varepsilon}\right)\right]
$$$$
\left.\times(-1)^{j-1}(2 \varepsilon-i) U_{j k, \varepsilon}\left(z_{j k}\right)\right\},
$$$$
(j=1,2) \text {, }
$$$$
k_{1}=\frac{k_{1}^{+}+k_{1}^{-}}{2}=\frac{1}{2} \sum_{j=1}^{2} \frac{\left(k_{1}^{+}\right)_{j}}{2}+\frac{1}{2} \sum_{j=1}^{2} \frac{\left(k_{1}^{-}\right)_{j}}{2},
$$$$
k=k_{2}+i k_{1}
$$

In order to express the change process of $z_{j k} \rightarrow a^{-}$ completely, it is necessary that the factor of (11): $\quad e^{(-1)^{j} \varepsilon \pi}(-1)^{j-1}\left[\sin \left(2 \varepsilon \ln \left(\left(\sqrt{a^{2}-x^{2}}\right) / x\right)\right) \quad-\right.$ $\left.i \cos \left(2 \varepsilon \ln \left(\left(\sqrt{a^{2}-x^{2}}\right) / x\right)\right)\right]=(-1)^{j-1}(2 \varepsilon-i),(\varepsilon \rightarrow 0)$ is substituted into the expressions (16b) and (17b). At the same time, the minus is used in (16c) because the load condition is given in (4).

Substituting (10), (7), (8), (15a), and (15b) into (16a)-(16c), (17a)-(17c), and (18), the stress intensity factors of interface crack for mode II can be deduced as

$$
\begin{gathered}
k_{2}=\tau(\pi a)^{1 / 2} p(\varepsilon), \quad k_{1}=-\tau(\pi a)^{1 / 2} \frac{2 g_{12} \varepsilon}{f_{12}} q(\varepsilon), \\
k=k_{2}+i k_{1}
\end{gathered}
$$

in which

$$
\begin{aligned}
p(\varepsilon)=\frac{1}{2}\{[ & +(1+2 \varepsilon) \operatorname{ch} \varepsilon \pi] \cos (\varepsilon \ln \pi a) \\
- & {[1+(1-2 \varepsilon) \operatorname{ch} \varepsilon \pi] \sin (\varepsilon \ln \pi a)\}, } \\
q(\varepsilon)=\frac{1}{2}\{[ & 1-(1+2 \varepsilon) \operatorname{ch} \varepsilon \pi] \cos (\varepsilon \ln \pi a) \\
- & {[1-(1-2 \varepsilon) \operatorname{ch} \varepsilon \pi] \sin (\varepsilon \ln \pi a)\} . }
\end{aligned}
$$

\section{Stress}

From (10), (8), and (7), it can be found as follows that

$$
\begin{array}{r}
\lim _{z_{j k} \rightarrow a}\left\{\left[2 \pi\left(z_{j k}-a\right)\right]^{(1 / 2)-i \varepsilon} U_{j k, \varepsilon}\left(z_{j k}\right)\right\}= \\
\tau(\pi a)^{(1 / 2)-i \varepsilon}, \\
(j, k=1,2)
\end{array}
$$

in the vicinity of the crack tip.

By (21), it can be known that

$$
\begin{array}{r}
U_{j k, \varepsilon}\left(z_{j k}\right)=\frac{\tau(\pi a)^{(1 / 2)-i \varepsilon}}{(2 \pi)^{(1 / 2)-i \varepsilon}} \frac{1}{\left(z_{j k}-a\right)^{(1 / 2)-i \varepsilon}}, \\
\left(z_{j k} \longrightarrow a ; j, k=1,2\right) .
\end{array}
$$

Substituting (22), (15a), (15b), (19), and (8) into (5a)-(5c), the stresses in the vicinity of the interface crack tip $\left(z_{j k} \rightarrow\right.$ $a, r \rightarrow 0 ; j, k=1,2)$ for mode II of orthotropic bimaterial can be expressed as follows:

$$
\begin{aligned}
\left(\sigma_{x}\right)_{j}= & \frac{k_{2}}{(2 \pi r)^{1 / 2}} \frac{\sqrt{2}}{\left(\beta_{j 2}-\beta_{j 1}\right) p(\varepsilon)} \\
& \times\left\{\left[\cos \left(\frac{\pi}{4}-\varepsilon \ln \pi a\right) \cdot \cos (\varepsilon \ln 2 \pi r)\right.\right. \\
& \left.\quad-\sin \left(\frac{\pi}{4}-\varepsilon \ln \pi a\right) \sin (\varepsilon \ln 2 \pi r)\right]
\end{aligned}
$$




$$
\begin{aligned}
& \cdot\left[\operatorname{Re} \frac{\beta_{j 1}^{2}}{\left(\cos \theta+i \beta_{j 1} \sin \theta\right)^{(1 / 2)-i \varepsilon}}\right. \\
& \left.-\operatorname{Re} \frac{\beta_{j 2}^{2}}{\left(\cos \theta+i \beta_{j 2} \sin \theta\right)^{(1 / 2)-i \varepsilon}}\right] \\
& -\left[\cos \left(\frac{\pi}{4}+\varepsilon \ln \pi a\right) \cos (\varepsilon \ln 2 \pi r)\right. \\
& \left.+\sin \left(\frac{\pi}{4}+\varepsilon \ln \pi a\right) \sin (\varepsilon \ln 2 \pi r)\right] \\
& \cdot\left[\operatorname{Im} \frac{\beta_{j 1}^{2}}{\left(\cos \theta+i \beta_{j 1} \sin \theta\right)^{(1 / 2)-i \varepsilon}}\right. \\
& \left.\left.-\operatorname{Im} \frac{\beta_{j 2}^{2}}{\left(\cos \theta+i \beta_{j 2} \sin \theta\right)^{(1 / 2)-i \varepsilon}}\right]\right\} \\
& +\frac{k_{1}}{(2 \pi r)^{1 / 2}} \frac{\sqrt{2} \beta_{j 1} \beta_{j 2}}{\left(\beta_{j 2}-\beta_{j 1}\right) q(\varepsilon)} \\
& \times\left\{\left[\cos \left(\frac{\pi}{4}+\varepsilon \ln \pi a\right) \cdot \cos (\varepsilon \ln 2 \pi r)\right.\right. \\
& \left.+\sin \left(\frac{\pi}{4}+\varepsilon \ln \pi a\right) \sin (\varepsilon \ln 2 \pi r)\right] \\
& {\left[\operatorname{Re} \frac{\beta_{j 2}}{\left(\cos \theta+i \beta_{j 2} \sin \theta\right)^{(1 / 2)-i \varepsilon}}\right.} \\
& \left.-\operatorname{Re} \frac{\beta_{j 1}}{\left(\cos \theta+i \beta_{j 1} \sin \theta\right)^{(1 / 2)-i \varepsilon}}\right] \\
& +\left[\cos \left(\frac{\pi}{4}-\varepsilon \ln \pi a\right) \cos (\varepsilon \ln 2 \pi r)\right. \\
& \left.-\sin \left(\frac{\pi}{4}-\varepsilon \ln \pi a\right) \sin (\varepsilon \ln 2 \pi r)\right] \\
& \cdot\left[\operatorname{Im} \frac{\beta_{j 2}}{\left(\cos \theta+i \beta_{j 2} \sin \theta\right)^{(1 / 2)-i \varepsilon}}\right. \\
& \left.\left.-\operatorname{Im} \frac{\beta_{j 1}}{\left(\cos \theta+i \beta_{j 1} \sin \theta\right)^{(1 / 2)-i \varepsilon}}\right]\right\}, \\
& \left(\sigma_{y}\right)_{j}=\frac{k_{2}}{(2 \pi r)^{1 / 2}} \frac{\sqrt{2}}{\left(\beta_{j 2}-\beta_{j 1}\right) p(\varepsilon)} \\
& \times\left\{\left[\cos \left(\frac{\pi}{4}-\varepsilon \ln \pi a\right) \cdot \cos (\varepsilon \ln 2 \pi r)\right.\right. \\
& \begin{array}{l}
\left.-\sin \left(\frac{\pi}{4}-\varepsilon \ln \pi a\right) \sin (\varepsilon \ln 2 \pi r)\right] \\
{\left[\operatorname{Re} \frac{1}{\left(\cos \theta+i \beta_{j 2} \sin \theta\right)^{(1 / 2)-i \varepsilon}}\right.} \\
\left.-\operatorname{Re} \frac{1}{\left(\cos \theta+i \beta_{j 1} \sin \theta\right)^{(1 / 2)-i \varepsilon}}\right] \\
-\left[\cos \left(\frac{\pi}{4}+\varepsilon \ln \pi a\right) \cos (\varepsilon \ln 2 \pi r)\right. \\
\left.+\sin \left(\frac{\pi}{4}+\varepsilon \ln \pi a\right) \sin (\varepsilon \ln 2 \pi r)\right]
\end{array} \\
& \cdot\left[\operatorname{Im} \frac{1}{\left(\cos \theta+i \beta_{j 2} \sin \theta\right)^{(1 / 2)-i \varepsilon}}\right. \\
& \left.\left.-\operatorname{Im} \frac{1}{\left(\cos \theta+i \beta_{j 1} \sin \theta\right)^{(1 / 2)-i \varepsilon}}\right]\right\} \\
& +\frac{k_{1}}{(2 \pi r)^{1 / 2}} \frac{\sqrt{2}}{\left(\beta_{j 2}-\beta_{j 1}\right) q(\varepsilon)} \\
& \times\left\{\left[\cos \left(\frac{\pi}{4}+\varepsilon \ln \pi a\right) \cdot \cos (\varepsilon \ln 2 \pi r)\right.\right. \\
& \left.+\sin \left(\frac{\pi}{4}+\varepsilon \ln \pi a\right) \sin (\varepsilon \ln 2 \pi r)\right] \\
& {\left[\operatorname{Re} \frac{\beta_{j 2}}{\left(\cos \theta+i \beta_{j 1} \sin \theta\right)^{(1 / 2)-i \varepsilon}}\right.} \\
& \left.-\operatorname{Re} \frac{\beta_{j 1}}{\left(\cos \theta+i \beta_{j 2} \sin \theta\right)^{(1 / 2)-i \varepsilon}}\right] \\
& +\left[\cos \left(\frac{\pi}{4}-\varepsilon \ln \pi a\right) \cos (\varepsilon \ln 2 \pi r)\right. \\
& \left.-\sin \left(\frac{\pi}{4}-\varepsilon \ln \pi a\right) \sin (\varepsilon \ln 2 \pi r)\right] \\
& \cdot\left[\operatorname{Im} \frac{\beta_{j 2}}{\left(\cos \theta+i \beta_{j 1} \sin \theta\right)^{(1 / 2)-i \varepsilon}}\right. \\
& \left.\left.-\operatorname{Im} \frac{\beta_{j 1}}{\left(\cos \theta+i \beta_{j 2} \sin \theta\right)^{(1 / 2)-i \varepsilon}}\right]\right\}, \\
& \left(\tau_{x y}\right)_{j}=\frac{k_{2}}{(2 \pi r)^{1 / 2}} \frac{\sqrt{2}}{\left(\beta_{j 2}-\beta_{j 1}\right) p(\varepsilon)}
\end{aligned}
$$




$$
\begin{aligned}
& \times\left\{\left[\cos \left(\frac{\pi}{4}+\varepsilon \ln \pi a\right) \cdot \cos (\varepsilon \ln 2 \pi r)\right.\right. \\
& \left.+\sin \left(\frac{\pi}{4}+\varepsilon \ln \pi a\right) \sin (\varepsilon \ln 2 \pi r)\right] \\
& {\left[\operatorname{Re} \frac{\beta_{j 2}}{\left(\cos \theta+i \beta_{j 2} \sin \theta\right)^{(1 / 2)-i \varepsilon}}\right.} \\
& \left.-\operatorname{Re} \frac{\beta_{j 1}}{\left(\cos \theta+i \beta_{j 1} \sin \theta\right)^{(1 / 2)-i \varepsilon}}\right] \\
& +\left[\cos \left(\frac{\pi}{4}-\varepsilon \ln \pi a\right) \cos (\varepsilon \ln 2 \pi r)\right. \\
& \left.-\sin \left(\frac{\pi}{4}-\varepsilon \ln \pi a\right) \sin (\varepsilon \ln 2 \pi r)\right] \\
& {\left[\operatorname{Im} \frac{\beta_{j 2}}{\left(\cos \theta+i \beta_{j 2} \sin \theta\right)^{(1 / 2)-i \varepsilon}}\right.} \\
& \left.\left.-\operatorname{Im} \frac{\beta_{j 1}}{\left(\cos \theta+i \beta_{j 1} \sin \theta\right)^{(1 / 2)-i \varepsilon}}\right]\right\} \\
& +\frac{k_{1}}{(2 \pi r)^{1 / 2}} \frac{\sqrt{2} \beta_{j 1} \beta_{j 2}}{\left(\beta_{j 2}-\beta_{j 1}\right) q(\varepsilon)} \\
& \times\left\{\left[\cos \left(\frac{\pi}{4}-\varepsilon \ln \pi a\right) \cdot \cos (\varepsilon \ln 2 \pi r)\right.\right. \\
& \left.-\sin \left(\frac{\pi}{4}-\varepsilon \ln \pi a\right) \sin (\varepsilon \ln 2 \pi r)\right] \\
& {\left[\operatorname{Re} \frac{1}{\left(\cos \theta+i \beta_{j 2} \sin \theta\right)^{(1 / 2)-i \varepsilon}}\right.} \\
& \left.-\operatorname{Re} \frac{1}{\left(\cos \theta+i \beta_{j 1} \sin \theta\right)^{(1 / 2)-i \varepsilon}}\right] \\
& -\left[\cos \left(\frac{\pi}{4}+\varepsilon \ln \pi a\right) \cos (\varepsilon \ln 2 \pi r)\right. \\
& \left.+\sin \left(\frac{\pi}{4}+\varepsilon \ln \pi a\right) \sin (\varepsilon \ln 2 \pi r)\right] \\
& {\left[\operatorname{Im} \frac{1}{\left(\cos \theta+i \beta_{j 2} \sin \theta\right)^{(1 / 2)-i \varepsilon}}\right.} \\
& \left.\left.-\operatorname{Im} \frac{1}{\left(\cos \theta+i \beta_{j 1} \sin \theta\right)^{(1 / 2)-i \varepsilon}}\right]\right\}
\end{aligned}
$$

\begin{tabular}{|c|c|c|c|c|c|c|c|}
\hline Materials & $\begin{array}{l}E_{j 1} / \\
\mathrm{GPa}\end{array}$ & $\begin{array}{l}E_{j 2} / \\
\mathrm{GPa}\end{array}$ & $v_{j}$ & $\begin{array}{l}\mu_{j} / \\
\mathrm{GPa}\end{array}$ & $\Delta_{j}$ & $\beta_{j 1}$ & $\beta_{j 2}$ \\
\hline$j=1$ & 12.45 & 11.56 & 0 & 4.35 & 0.54 & 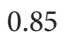 & 1.21 \\
\hline$j=2$ & 201 & 12.5 & 0 & 6.69 & 813.31 & 0. & 5.39 \\
\hline$B \quad j=1$ & 181 & 10.3 & & 7.17 & 539.01 & 0.86 & 4.89 \\
\hline${ }^{D} \quad j=2$ & 40.06 & 38.26 & 0.26 & 11.24 & 5.07 & 0.63 & 1.63 \\
\hline$j=$ & 18.3 & 15.51 & 0.32 & 5.87 & 1.4 & 0.80 & 1.35 \\
\hline$j=$ & 135 & 10.3 & 0.21 & 6.6 & 348.96 & 0.82 & 4.40 \\
\hline
\end{tabular}

in which $p(\varepsilon)$ and $q(\varepsilon)$ are given by (20a) and (20b).
TABLE 1: Mechanical properties of each orthotropic material.

TABLE 2: Mechanical properties of three orthotropic bimaterials.

\begin{tabular}{lcccccc}
\hline Bimaterial & $e_{12}$ & $f_{12}$ & $g_{12}$ & $h_{12}$ & $\varepsilon$ & $\lambda$ \\
\hline$A$ & 0.0804 & 0.0379 & 0.1966 & 0.2948 & -0.0834 & -0.5 \\
& & & & & & $-0.0834 i$ \\
$B$ & 0.0505 & 0.0026 & 0.0881 & 0.1908 & -0.0427 & -0.5 \\
& & & & & & $-0.0427 i$ \\
& & & & & & \\
C & 0.0188 & 0.0166 & 0.1565 & 0.2681 & -0.0149 & $-0.0149 i$ \\
\hline
\end{tabular}

Note that (23a)-(23c) of the stress field contain the following terms:

$$
\left(\begin{array}{c}
\left(\sigma_{x}\right)_{j} \\
\left(\sigma_{y}\right)_{j} \\
\left(\tau_{x y}\right)_{j}
\end{array}\right) \supset \frac{1}{(2 \pi r)^{1 / 2}}\left(\begin{array}{c}
\cos (\varepsilon \ln 2 \pi r) \\
\sin (\varepsilon \ln 2 \pi r)
\end{array}\right) .
$$

The right side of (24) tends to change the sign rapidly at the crack tip $(r \rightarrow 0)$, and therefore the stresses possess an oscillatory character.

\section{Oscillatory Field}

The test and calculus results [16, 22-24] for the mechanicals properties of three orthotropic bimaterial are shown in Tables 1 and 2.

From (19), the normalized stress intensity factors $k_{2} / \tau$ and $k_{1} / \tau$ depend on the length of crack $a$ and the bielastic constant $\varepsilon$. The variations of the stress intensity factors versus crack length are plotted in Figure 2. The variations of the stress intensity factors with bielastic constant are illustrated in Figure 3. The minute variations of factor $k_{1} / \tau$ can be observed by the inner small figure of Figure 3. The factors $k_{2} / \tau$ and $k_{1} / \tau$ increase almost linearly when the length $a$ or the constant $\varepsilon$ increases as shown in Figures 2 and 3 .

By (23a)-(23c), the normalized stresses $\sigma_{x} / \tau, \sigma_{y} / \tau$, and $\tau_{x y} / \tau$ depend on the polar angle $\theta$ and the polar radius $r$. The variations of the normalized stresses for the above three bimaterials with respect to polar angle $\theta$ are plotted in Figure 4 . Figure 4 shows that three stresses have alternately the increase and decrease with $\theta$ increase, their maximum values can be always reached at both sides of the crack.

Figures 5 and 6 show the variations of the normalized stresses for the above three bimaterials with respect to polar radius $r$ for $\theta= \pm 45^{\circ}$ and $\theta= \pm 60^{\circ}$. Two figures mean that the stresses $\sigma_{x} / \tau, \sigma_{y} / \tau$, and $\tau_{x y} / \tau$ are the monotonic decreasing functions of $r / a$ on the two half-planes. 


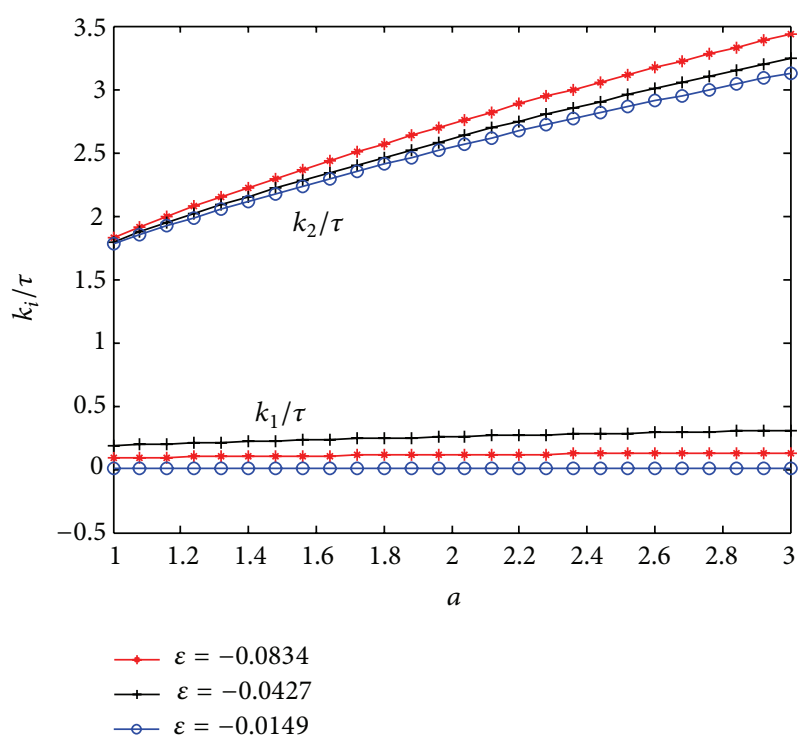

FIgURE 2: Normalized stress intensity factors as a function of $a$.

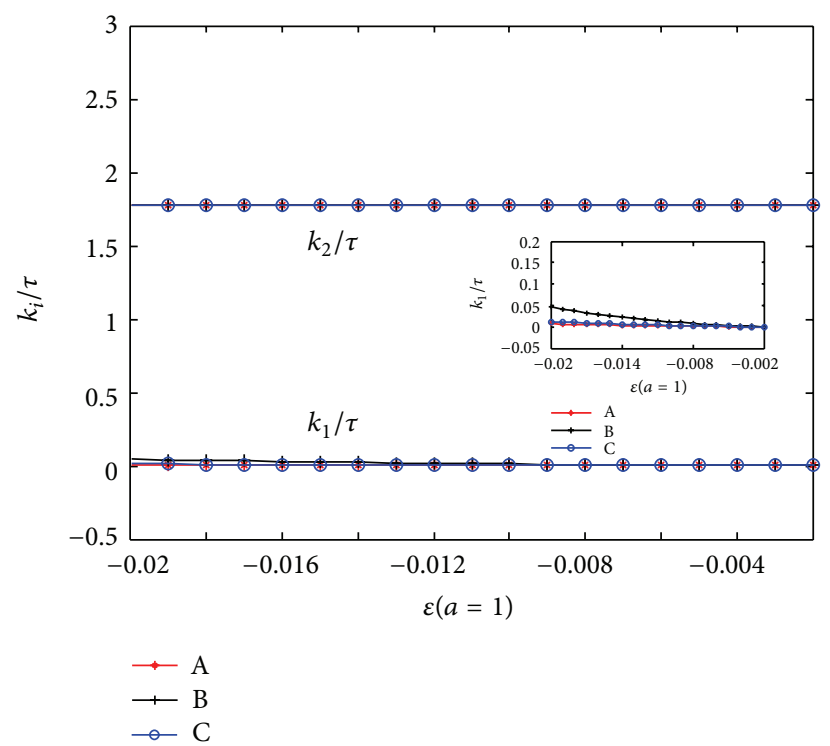

FIGURE 3: Normalized stress intensity factors as a function of $\varepsilon$ when $a=1$.

Figures 7 and 8 show the variations of the normalized logarithmic stresses with respect to the polar radius $r$ for $\theta=$ $\pm 45^{\circ}$ and $\theta= \pm 60^{\circ}$. It can be found that the stress distribution in Figures 7 and 8 is not straight line and does not parallel to each other. Such distributions mean oscillatory singularity state.

\section{Conclusion}

From the above derivation, the following results are very significant.

(1) New stress functions are chosen.

(2) The system of twelve nonhomogeneous linear equations is derived.
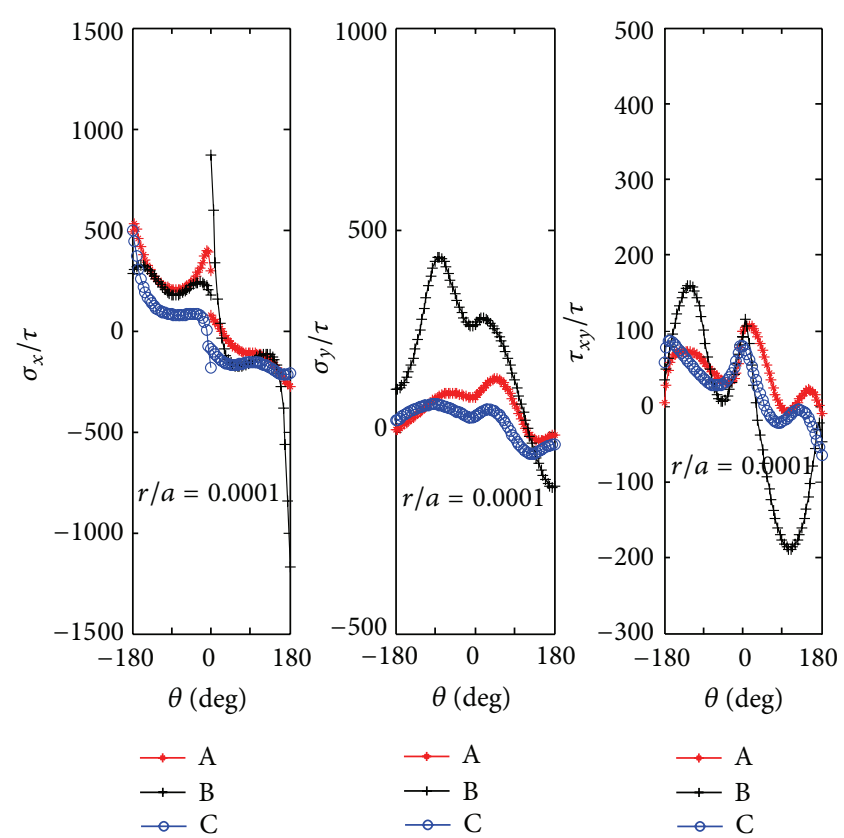

FIGURE 4: Normalized stresses versus $\theta$ for $r / a=0.0001$.
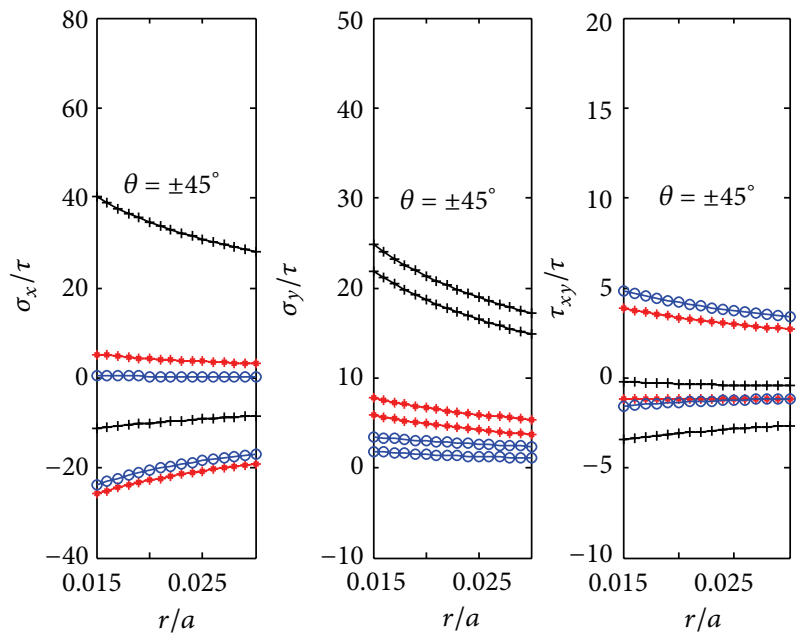

$$
\begin{aligned}
& \rightarrow \mathrm{A} \\
& \div \mathrm{B} \\
& \rightarrow \mathrm{C}
\end{aligned}
$$$$
\begin{aligned}
& \rightarrow \mathrm{A} \\
& \rightarrow \mathrm{B} \\
& \rightarrow \mathrm{C}
\end{aligned}
$$$$
\begin{aligned}
& \rightarrow \mathrm{A} \\
& \rightarrow \mathrm{B} \\
& \rightarrow \mathrm{C}
\end{aligned}
$$

FIGURE 5: Normalized stresses versus $r$ for $\theta= \pm 45^{\circ}$.

(3) The characteristic equation can be given.

(4) By the help of the distinction rule $(*)$, the complex singularity exponent $\lambda$ can be found.

(5) Total coefficients are determined.

(6) For the oscillatory field, the theoretical formulae of stress intensity factors and the analytic solutions for stresses near interface crack tip of mode II are 


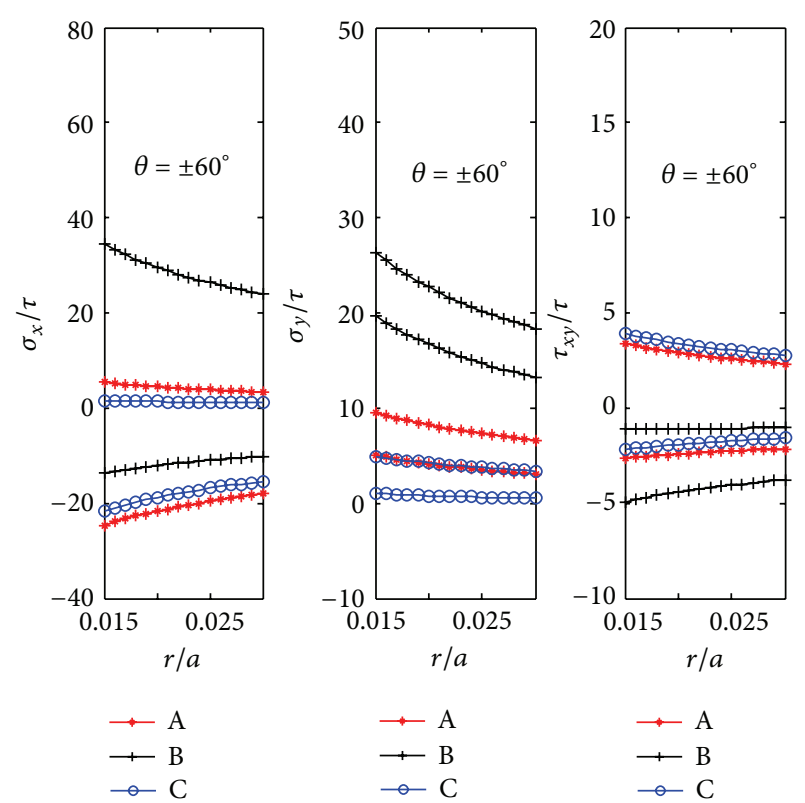

Figure 6: Normalized stresses versus $r$ for $\theta= \pm 60^{\circ}$.

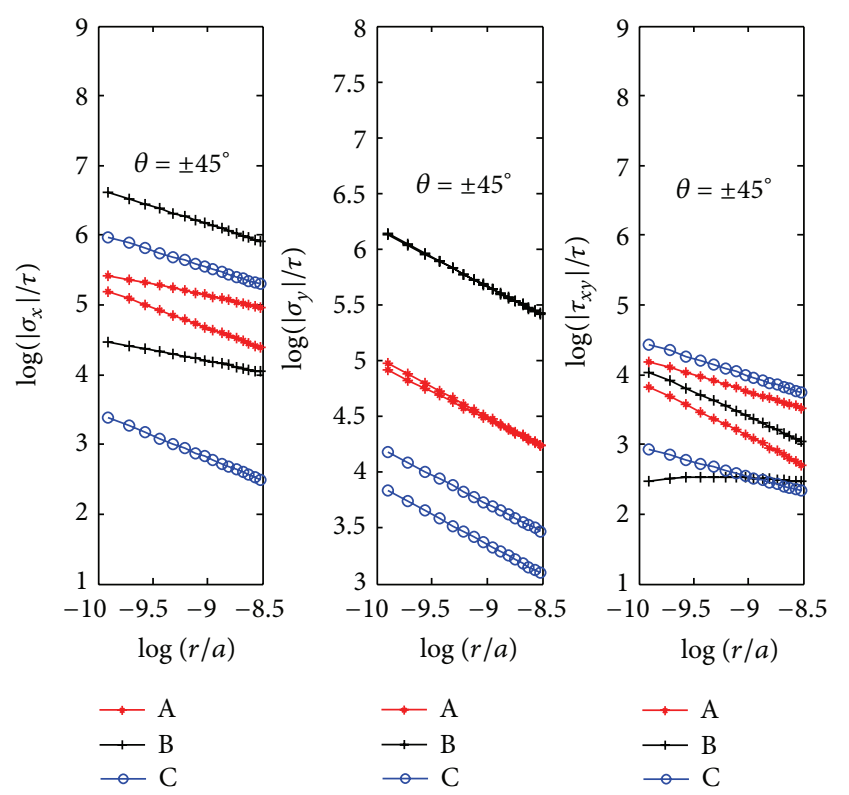

FIGURE 7: Normalized stresses versus $r$ for $\theta= \pm 45^{\circ}$.

yielded, respectively. The stress intensity factors and the stresses show mixed crack characteristics.

(7) The crack tip fields for oscillatory singularities are illustrated in Figures 2 to 8.

\section{Acknowledgment}

This work was supported by the Natural Science Foundation of Shanxi province (no. 2011011021-3), and the doctoral fund of Taiyuan University of Science and Technology (no. 20102028).
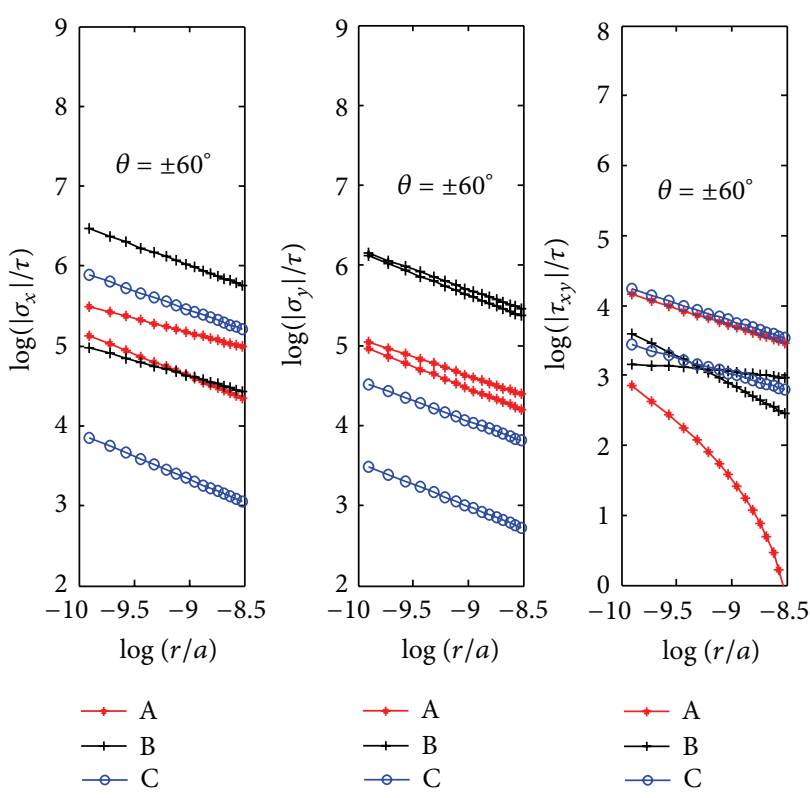

Figure 8: Normalized stresses versus $r$ for $\theta= \pm 60^{\circ}$.

\section{References}

[1] M. L. Williams, "The stresses around a fault or crack in dissimilar media," Bulletin of the Seismological Society of America, vol. 49, no. 2, pp. 199-204, 1959.

[2] J. R. Rice and G. C. Sih, "Plane problems of cracks in dissimilar media," Journal of Applied Mechanics, vol. 32, no. 3, pp. 418-423, 1965.

[3] F. Erdogan, "Stress distribution in bonded dissimilar materials with cracks," Journal of Applied Mechanics, vol. 32, no. 3, pp. 403-410, 1965.

[4] A. H. England, "A crack between dissimilar media," Journal of Applied Mechanics, vol. 32, no. 3, pp. 400-402, 1965.

[5] K. Y. Lin and J. W. Mar, "Finite element analysis of stress intensity factors for cracks at a bi-material interface," International Journal of Fracture, vol. 12, no. 4, pp. 521-531, 1976.

[6] Z. Suo, "Singularities, interfaces and cracks in dissimilar anisotropic media," Proceedings of the Royal Society of London Series A, vol. 427, no. 1873, pp. 331-358, 1990.

[7] J. Chang and J.-Q. Xu, "The singular stress field and stress intensity factors of a crack terminating at a bimaterial interface," International Journal of Mechanical Sciences, vol. 49, no. 7, pp. 888-897, 2007.

[8] D. Ying and J. Xing, "Researches on stress singulality of interface end and distributive law of interface stress," Science in China, G Series, vol. 37, no. 4, pp. 535-543, 2007 (Chinese).

[9] X. S. Zhang, "A central crack at the interface between two different orthotropic media for the mode I and mode II," Engineering Fracture Mechanics, vol. 33, no. 3, pp. 327-333, 1989.

[10] Z. Suo and J. W. Hutchinson, "Interface crack between two elastic layers," International Journal of Fracture, vol. 43, no. 1, pp. 1-18, 1990.

[11] H. Gao, M. Abbudi, and D. M. Barnett, "Interfacial crack-tip field in anisotropic elastic solids," Journal of the Mechanics and Physics of Solids, vol. 40, no. 2, pp. 393-416, 1992. 
[12] F. Erdogan and B. Wu, "Interface crack problems in layered orthotropic materials," Journal of the Mechanics and Physics of Solids, vol. 41, no. 5, pp. 889-917, 1993.

[13] K.-P. Ma and C.-T. Liu, "Semi-weight function method on computation of stress intensity factors in dissimilar materials," Applied Mathematics and Mechanics, vol. 25, no. 11, pp. 12411248, 2004.

[14] L. Marsavina and T. Sadowski, "Stress intensity factors for an interface kinked crack in a bi-material plate loaded normal to the interface," International Journal of Fracture, vol. 145, no. 3, pp. 237-243, 2007.

[15] Y. Chen, P. Z. Qiao, H. D. Jiang, and Q. W. Ren, "Review on experimental methods and fracture models for bimaterial interfaces," Advances in Mechanics, vol. 38, no. 1, pp. 53-61, 2008 (Chinese).

[16] G. C. Sih and E. P. Chen, Cracks in Composite Materials, vol. 6 of Mechanics of Fracture, Martinus Nijhoff, The Hague, The Netherlands, 1981.

[17] G. C. Sih and H. Liebowitz, "Mathematical theories of brittle fracture," in Fracture, H. Liebowitz, Ed., vol. 2, pp. 89-131, Academic Press, New York, NY, USA, 1971.

[18] H. T. Corten, "Fracture mechanics of composites," in Fracture, H. Liebowitz, Ed., vol. 7, pp. 695-703, Academic Press, New York, NY, USA, 1972.

[19] S. G. Lekhnitskii, Theory of Elasticity of an Anisotropic Elastic Body, Science Press, Beijing, China, 1963.

[20] W.-y. Yang, J.-1. Li, and X.-x. Zhang, Method of a Complex variable for fracture in Composite Materials, Science Press, Beijing, China, 2005.

[21] Department of mathematics, Beijing University, Advanced Algebra, Advanced Education Press, Beijing, China, 2002.

[22] S. Q. Zhang and W.-Y. Yang, "Prediction of mode I crack propagation direction in carbon-fiber reinforced composite plate," Applied Mathematics and Mechanics, vol. 25, no. 6, pp. 714-722, 2004.

[23] J.-L. Li, G.-L. Feng, B.-B. Chen, and J. Zhang, "Application of complex method in the fracture problem of bimaterial," Journal of North University of China, vol. 32, no. 1, pp. 104-107, 2011 (Chinese).

[24] J.-1. Li and X.-1. Wang, "Interface end stress field of antiplane of orthotropic bimaterials," Applied Mathematics and Mechanics, vol. 30, no. 9, pp. 1153-1159, 2009. 


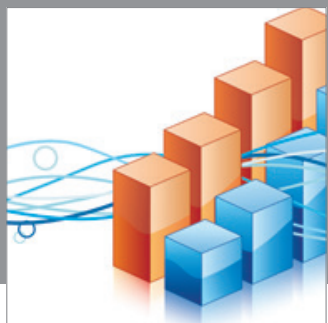

Advances in

Operations Research

mansans

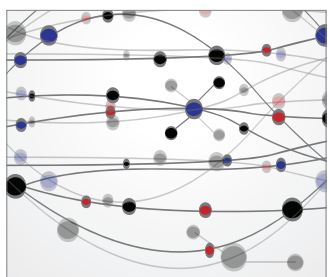

The Scientific World Journal
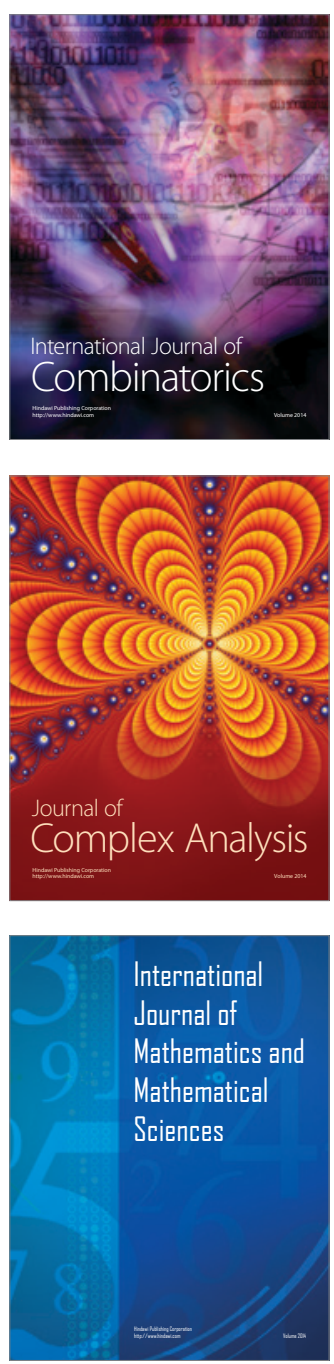
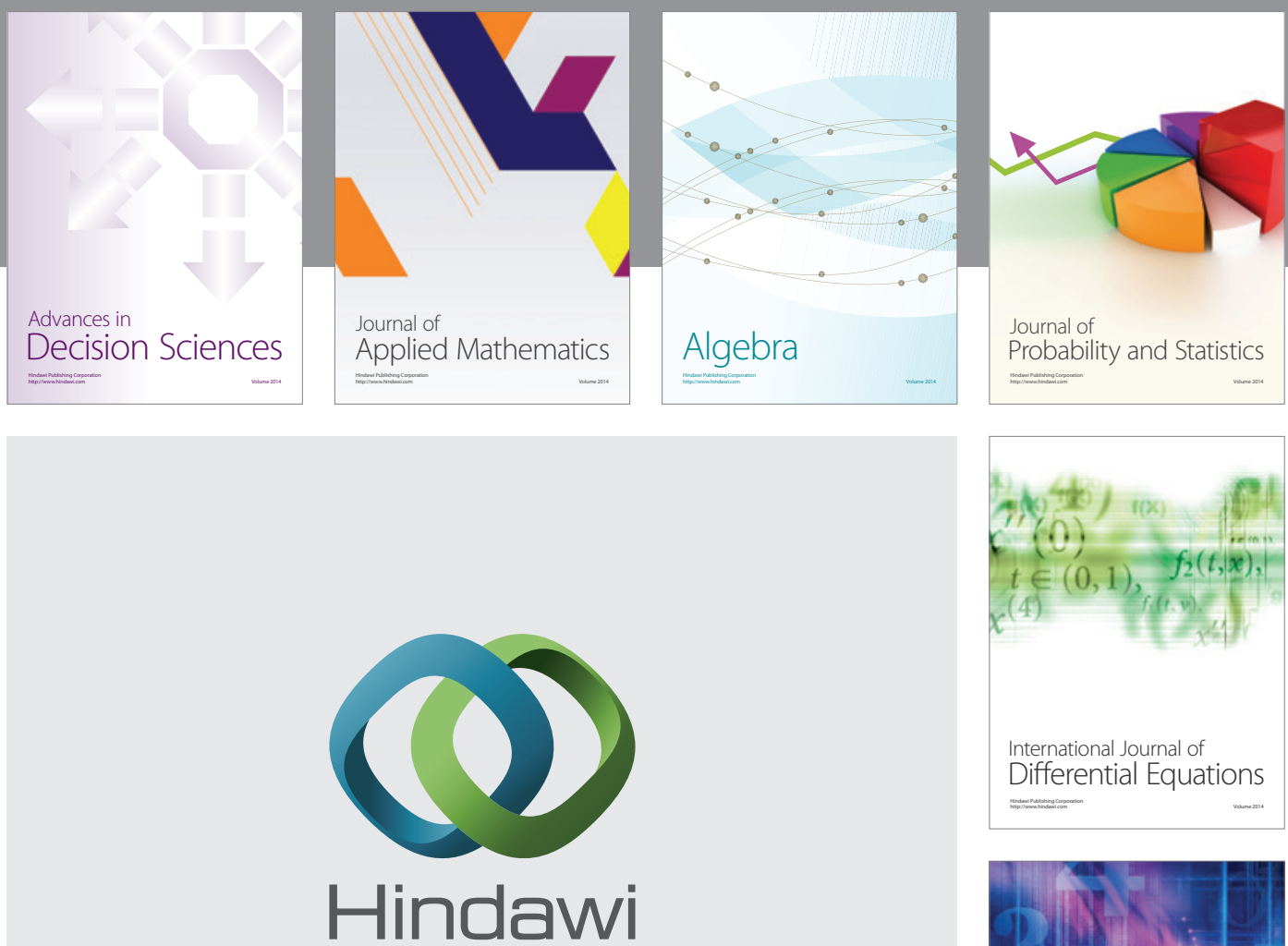

Submit your manuscripts at http://www.hindawi.com
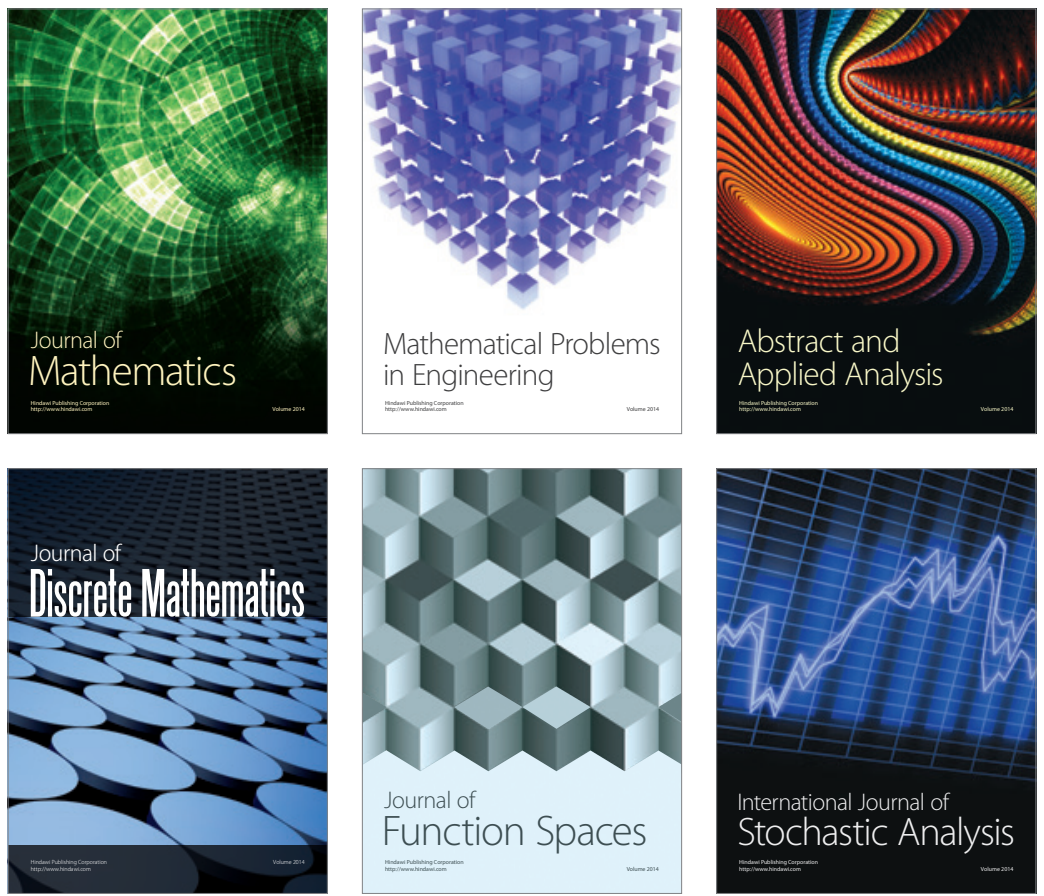

Journal of

Function Spaces

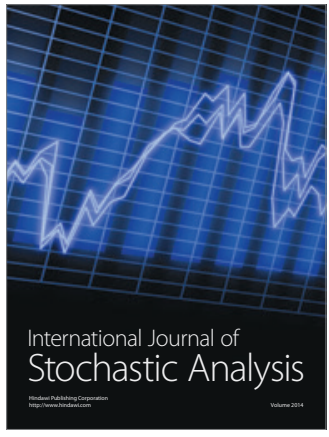

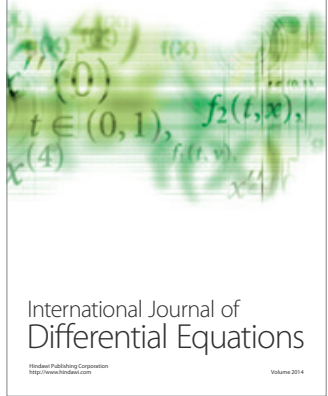
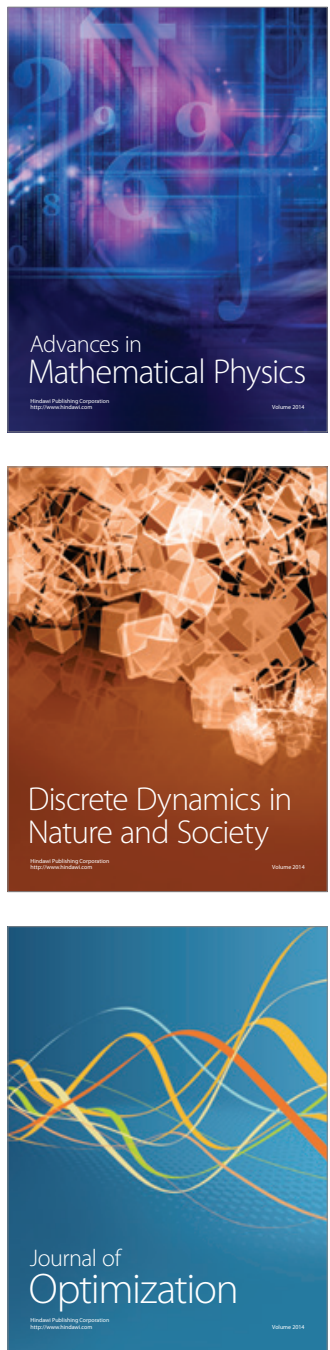